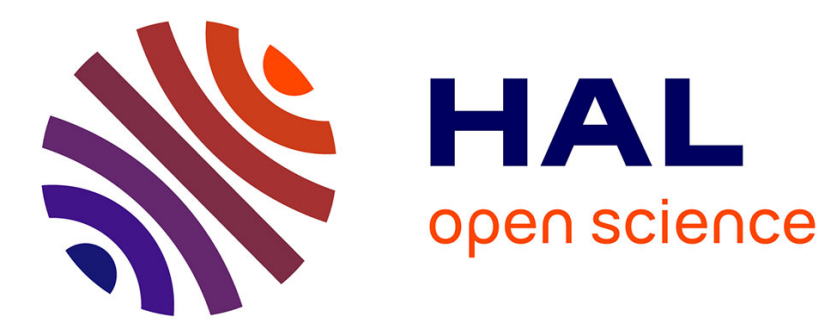

\title{
Simulation of flank milling processes
}

\author{
Arnaud Larue, Yusuf Altintas
}

\section{To cite this version:}

Arnaud Larue, Yusuf Altintas. Simulation of flank milling processes. International Journal of Machine Tools and Manufacture, 2005, 45 (4-5), pp.549-559. 10.1016/j.ijmachtools.2004.08.020 . hal00098357

\section{HAL Id: hal-00098357 \\ https://hal.science/hal-00098357}

Submitted on 25 Feb 2018

HAL is a multi-disciplinary open access archive for the deposit and dissemination of scientific research documents, whether they are published or not. The documents may come from teaching and research institutions in France or abroad, or from public or private research centers.
L'archive ouverte pluridisciplinaire HAL, est destinée au dépôt et à la diffusion de documents scientifiques de niveau recherche, publiés ou non, émanant des établissements d'enseignement et de recherche français ou étrangers, des laboratoires publics ou privés. 


\title{
Simulation of flank milling processes
}

\author{
A. Larue*, Y. Altintas \\ Manufacturing Automation Laboratory, Department of Mechanical Engineering, The University of British Columbia, \\ 2324 Main Mall, Vancouver, BC, Canada V6T 1Z4
}

The paper presents prediction of cutting forces when flank milling ruled surfaces with tapered, helical, ball end mills. The geometric model of the workpiece is imported from standard CAD systems, and the tapered helical ball end mill is modeled as the combination of sphere and cone primitives in $\mathrm{ACIS}^{\odot}$ solid modeling environment. The intersection of cutter and part with a ruled surface is evaluated, and the cutter entry into and exit angles from the work material are modeled, and stored as a function of tool center coordinates along the path. The cutter entry and exit angles, the immersion angles, are used as boundary conditions in predicting the cutting forces along the path. The methodology allows prediction of cutting load distribution on the tool and part, as well optimization of machining cycle times by scheduling the feedrate in such a way that torque, power and static deflections can be maintained at safe levels.

Keywords: Flank milling; Feedrate optimization; Various cutter workpiece engagement

\section{Introduction}

Flank milling operations are common in aerospace industry, especially when milling impellers and blades of the gas turbines. The operations are usually conducted on three-, four- and five-axis machine tools depending on the complexity of the airfoils to be machined. The part is modeled and multi-axis NC tool path is generated in CAD environment. However, the machining process physics is not considered in selecting feeds, speeds and depth of cuts during NC tool path generation step. This paper presents a method which integrates process simulation and optimization to flank milling operations conducted with tapered helical ball end mills.

Majority of the past research in flank milling has been dedicated to modeling of the ruled surface which can be machined in flank milling mode. Wu showed that a blade surface can be flank milled if it can be approximated by a ruled surface [1]. Wu also showed that it is even possible to

\footnotetext{
* Corresponding author. Tel.: + 1604827 5007; fax: +1 6048222432

E-mail addresses: larue@mech.ubc.ca (A. Larue), http://www.mech. ubc.ca/ mal (A. Larue).
}

flank mill more complex surfaces at several passes, as long as each pass can be aligned with a partial rules surface found on the blades. The second challenge is to position the tool in such a way that it does not interfere with the final surface of the blade (Fig. 1a), while keeping the tool trajectory smooth (Fig. 1b) [2]. High speed machining (HSM) requires taking into account of the dynamical properties of recent numerical controller during the tool path generation stage. Chiou et al. presents a swept envelope approach to determine tool position for five-axis machining $[3,4]$. He presents a closed form solution of the swept profile, and shows 3D shapegenerating profiles for generalized cutter shapes [5]. Lartigue et al. [6] developed a method for deforming the top and bottom B-spline curves of the surface in order to minimize the geometric machining errors. Tonshoff et al. [7] and Monies et al. [8] also presented methods to minimize the geometric machining errors, but by manipulating the tool position as opposed to splines of the ruled surface.

Once the tool path generation stage is performed, chatter vibrations and static tool deflections during flank milling process have to be controlled. Indeed, cutting forces vary due to the various engagements of the cutter in the workpiece (Fig. 2), and can lead to geometrical errors on the machined part. 


\begin{tabular}{|c|c|}
\hline \multicolumn{2}{|l|}{ Nomenclature } \\
\hline $\begin{array}{l}\text { tool radius when the plane of cut related to the } \\
\text { top curve of the ruled surface }\end{array}$ & $\begin{array}{l}\text { pitch_dis pitch of discretization for immersion angles } \\
N_{\mathrm{f}} \quad \text { number of flutes }\end{array}$ \\
\hline radius of the tool's ball nose & $f_{z} \quad$ average feedrate per tooth \\
\hline half taper angle of the tool & flute number $\left(p=0,1, \ldots, N_{\mathrm{f}}-1\right)$ \\
\hline number of rules on the surface to be machined & $F_{\mathrm{t} p}, F_{\mathrm{r} p}, F_{\mathrm{a} p}$ tangential, radial and axial force acting on \\
\hline number of planes of cut in $z$-direction & $p$ th flute \\
\hline knot number along the ruled surface $\left(j=0,1, \ldots, N_{\mathrm{r}}-1\right)$ & $F_{x}, F_{y}, F_{z}$ force components in $X, Y$ and $Z$ direction \\
\hline index number of the plane of cut $\left(i=0,1, \ldots, N_{i}-1\right)$ & $\mathrm{d} S \quad$ differential cutting edge length \\
\hline $\begin{array}{l}\text { unit normal vector of the machined surface at } \\
\text { point } B_{j}\end{array}$ & $\begin{array}{l}\text { projected length of an infinitesimal cutting flute } \\
\text { in direction along the cutting velocity }\end{array}$ \\
\hline$\vec{n}_{T j} \quad$ unit normal vector of the machined surface at & differential time interval for digital integration \\
\hline $\begin{array}{ll}\overrightarrow{k_{B j}}=v & \text { unit vector of the rule }\end{array}$ & $\begin{array}{l}\text { differential angular rotation for digital } \\
\text { integration }\end{array}$ \\
\hline $\begin{array}{ll}\overrightarrow{t_{B j}}=u & \text { unit tangent vector of the bottom curve at point } \\
& B_{j} \text { representing the machining direction }\end{array}$ & $\begin{array}{ll}\mathrm{d} r & \text { differential radius for digital integration } \\
\kappa, \kappa_{p} & \text { angle between cutting edge normal and } Z \text {-axis }\end{array}$ \\
\hline $\begin{array}{ll}T_{O}, u, v, w & \text { local coordinate system } \\
\mathrm{d} z \quad \text { uniform differential height }\end{array}$ & $\begin{array}{l}K_{\mathrm{tc}}, K_{\mathrm{rc}}, K_{\mathrm{ac}} \text { cutting force coefficients in tangential, } \\
\text { radial and axial directions }\end{array}$ \\
\hline$z_{i} \quad$ height of the $i$ th plane of cut & $K_{\mathrm{te}}, K_{\mathrm{re}}, K_{\mathrm{ae}}$ edge force coefficients in tangential, radial \\
\hline height of the $i+1$ th plane of cut & and axial directions \\
\hline unit vector representing the tool axis & angular velocity of the spindle $(\Omega=2 \pi n / 60), n$ \\
\hline unit vector perpendicular to the unit tool axis & being the spindle speed in rpm \\
\hline vector and to the unit feed vector at point $A_{i j}$ & $\phi_{p} \quad$ rotation angle of cutting edge $p$ \\
\hline$\phi_{S_{\text {Sccalculated }}}$ effective calculated entry angle & $r(z), r_{i}$ radius of the cutter at $z$ level (ith plane of cut) \\
\hline $\begin{array}{l}\phi_{\text {e_calculated }} \text { effective calculated exit angle } \\
\phi_{\text {start }}, \phi_{\text {exit }} \text { entry and exit angles of cut }\end{array}$ & $\begin{array}{l}\phi \quad \text { total angular rotation for cutting edge on the } X Y \\
\text { plane }\end{array}$ \\
\hline $\begin{array}{l}\text { Abcurv } v_{x} \text { curvilinear abscissa in } u \text {-direction (machining } \\
\text { direction) }\end{array}$ & $z_{\text {start }}(\phi), z_{\text {exit }}(\phi)$ axial integration limits \\
\hline
\end{tabular}

There has been very little research reported in predicting the cutting forces during flank milling. The cutting force prediction allows identification of loads on the blades during machining, process planning of the machining process to avoid excessive loading of the spindle drive, and it is used as a basis to avoid chatter vibrations [9]. Ramaraj presented the geometry of the tapered helical ball end mill which can be used in predicting the chip load distribution; hence the cutting forces [10]. Altintas et al. [11,12] presented generalized mathematical models which lead to the prediction of cutting forces for any end mill, including tapered helical ball end mills used in flank milling of blades. However, the papers show prediction of cutting forces when the immersion angles are known and the process is stationary. Budak presented process simulation and adaptive machining of impeller as a whole, while considering the force and chatter constraints [13]. He measured the cutting forces, linearized them, and scheduled feedrates along the tool path in order to maintain the load on the cutter and blade at the desired level.

This article presents an alternative to on-line process optimization. It is proposed that the machining forces can be (a)

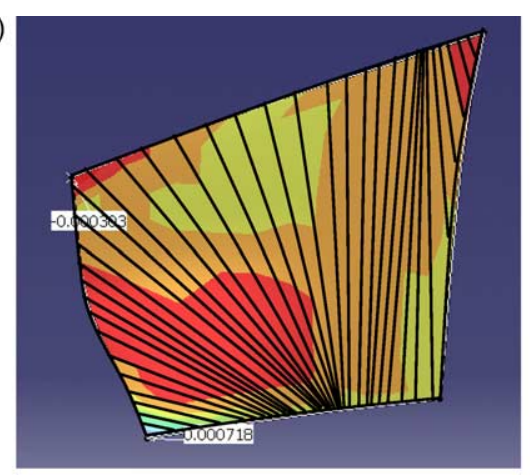

(b)

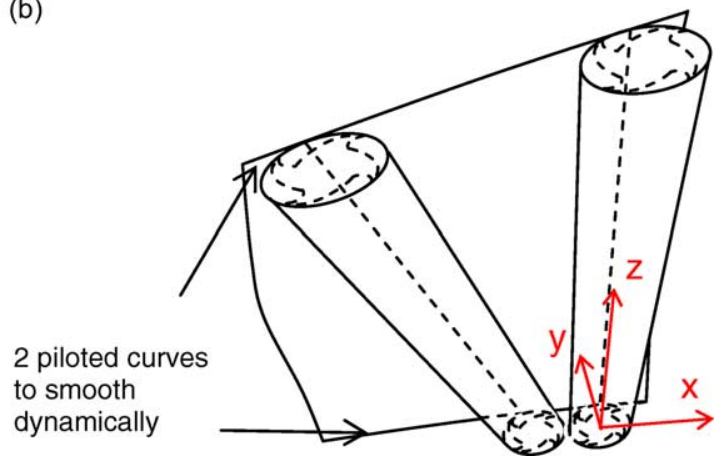

Fig. 1. Tool path generation for five-axis flank milling of impellers. 


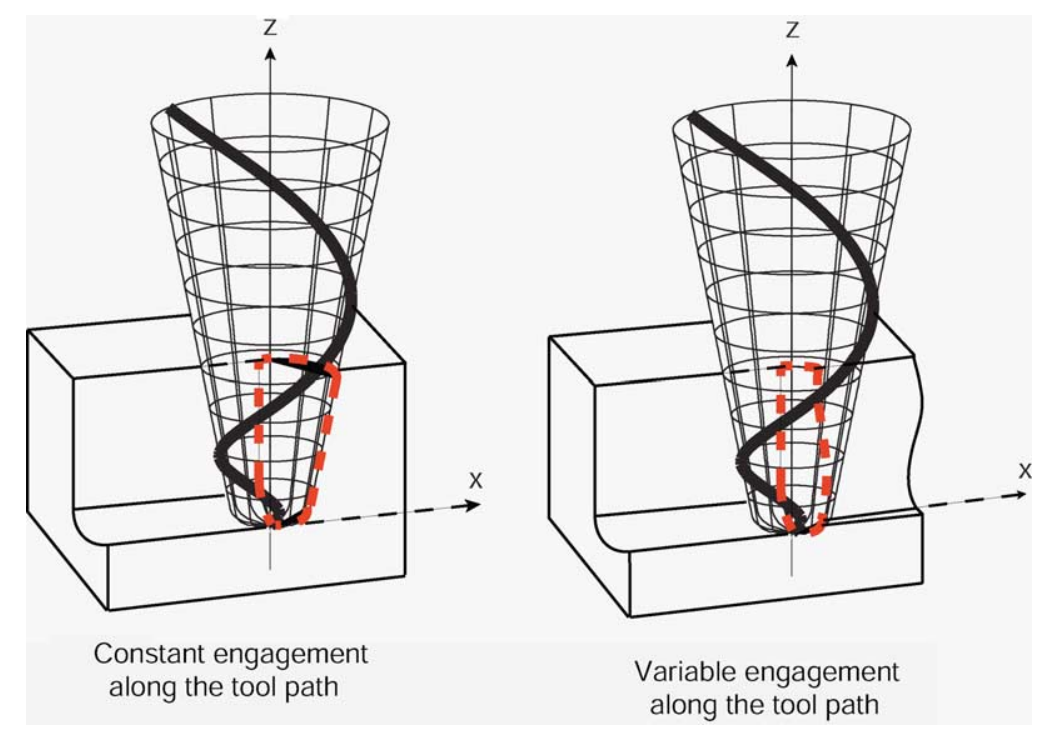

Fig. 2. Variable cutter/workpiece engagement.

predicted in virtual environment by modeling the cutter/part intersection along the tool path, and the feed can be scheduled.

Henceforth, the paper is organized as follows. The tool position is analytically modeled, and an algorithm to evaluate immersion angles from cutter/part intersection is presented in Section 2. The part and tool path are brought from CAD system, and the intersections are evaluated in ACIS $^{\odot}$ solid modeling environment [14]. The prediction of cutting forces are presented in Section 3, followed by experimental verification of flank milling of sample ruled surface in Section 4. The paper is concluded in Section 5.

\section{Prediction of immersion angles}

The prediction of cutting forces requires identification of cutter/workpiece intersection boundaries along the tool path when milling ruled surfaces such as flank milling of impellers. The workpiece geometry is assumed to be modeled in a CAD system, and its geometric model is imported using IGES or STEPNC standards. In addition, the NC program can also be imported in the form of industry standard cutter location (CL) file. The flank milling requires orientation of tapered helical ball end mill in such a way that its periphery is tangent to a straight line which connects the upper and lower splines of the ruled surface.

The part is represented by a ruled surface whose parameters are provided in the CAD model. The tapered helical ball end mill is modeled as a combination of a cone and sphere. Both parts are defined in Cartesian coordinate system. Although the concept can be extended to five-axis flank milling of impellers, this paper presents three-axis peripheral milling of a part with ruled outer surface. The immersion history of tapered helical ball end when milling a ruled surface is formulated by modeling tool location, intersection of tool and workpiece with ruled surface, and immersion history along the cutter axis.

\subsection{Modeling of cutter location}

Consider flank milling of a ruled surface as given in Fig. 3 . The top and bottom curves are represented by any parametric spline which is partitioned with equal number of knots. The corresponding knots on the upper and lower curves form straight lines which define the ruled surface with uniform number of patches. The cutter is divided into a number of discrete disc elements along its axis $\vec{E}_{j} \vec{C}_{T j}$ where $j$ represents the knot number along the ruled surface. Each disc is like a circle with varying radius along the tapered helical ball end mill axis. The periphery of the cutter, or each circle along the cutter axis, is tangent to the ruled surface at points $B_{j}$ and $T_{j}$ when the tool is cutting the patch $j$. The cutter's tip is at $E_{j}$, the center of the ball end is at $C_{B j}$. The cutter body and part are defined using a common coordinate system $O(\vec{X}, \vec{Y}, \vec{Z})$. The rule surface normals at $B_{j}$ and $T_{j}$ are given in the CAD model as $\overrightarrow{n_{B j}}$ and $\overrightarrow{n_{T j}}$, respectively. However, they are assumed to be equal here for the simplicity of the immersion calculation. The tool location is defined by the tool tip $E_{j}\left(\overrightarrow{O E}_{j}\right)$ and cutter axis $\overrightarrow{C_{B j}} C_{T j}$

$\overrightarrow{O E_{j}}=O \vec{C}_{B j}-r_{b} \cdot(1+\tan (\gamma)) \cdot \frac{\overrightarrow{C_{B j} C_{T j}}}{\left\|\overrightarrow{C_{B j} C_{T j}}\right\|}$

where the tool direction is given by ${\overrightarrow{C_{B j}}}_{C_{T j}}=O \vec{C}_{T j}-O \vec{C}_{B j}$, and position vectors $O \vec{C}_{B j}$ and $O \vec{C}_{T j}$ are evaluated from

$\left.\begin{array}{l}\overrightarrow{O C_{B j}}=\overrightarrow{O B_{j}}+\vec{B}_{j} \vec{C}_{B j}=\overrightarrow{O B_{j}}+\frac{r_{\mathrm{b}}}{\cos (\gamma)} \cdot \vec{n}_{B j} \\ O \overrightarrow{C C}_{T j}=\overrightarrow{O T}_{j}+\vec{T}_{j} \vec{C}_{T j}=\overrightarrow{O T}_{j}+\frac{r_{\mathrm{t}}}{\cos (\gamma)} \cdot \vec{n}_{B j}\end{array}\right\}$

where $r_{\mathrm{b}}$ and $\gamma$ are the ball radius and half cone angle of the tapered ball end mill, see Fig. 3 . The vectors $\overrightarrow{O B}_{j}$ and $\overrightarrow{O T}_{j}$ in 


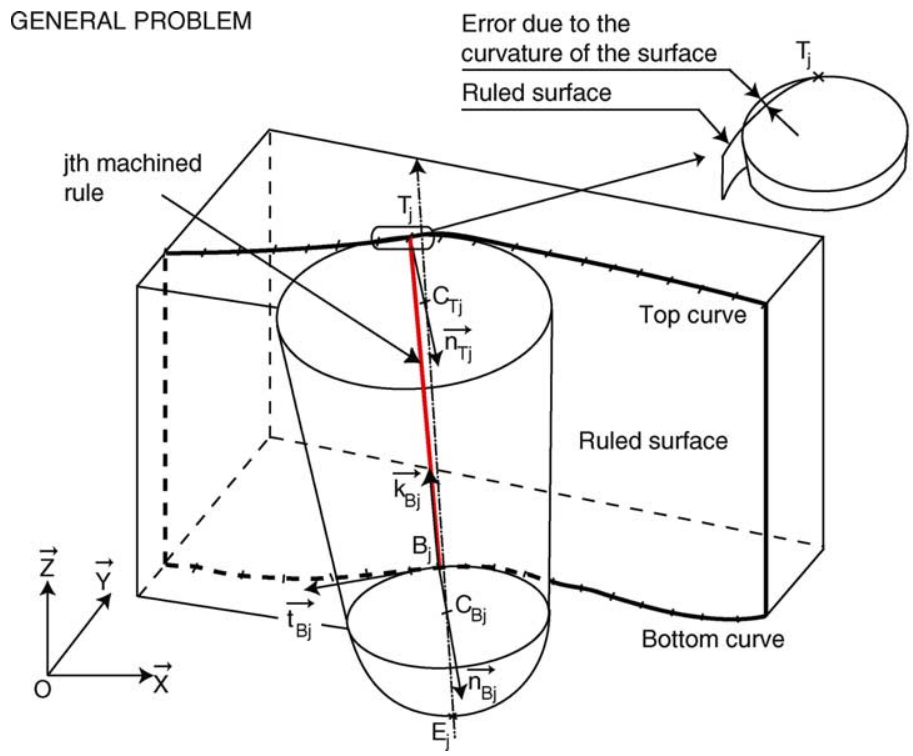

Fig. 3. Tool positioning along a given ruled surface.

Eq. (2) are given in the rule surface model since they are on patch $j$. The radius of the cutter $\left(r_{\mathrm{t}}\right)$ when it is tangent to the surface at point $T_{j}$ can be expressed from the ruled surface as

$r_{\mathrm{t}}=r_{\mathrm{b}}+\sin (\gamma) \cdot \| \overrightarrow{B_{j}} T_{j}$

where $\overrightarrow{B_{j} T_{j}}=\overrightarrow{O T_{j}}-\overrightarrow{O B_{j}}$. The unit normal $\left(\overrightarrow{n_{B j}}\right)$ of the ruled surface at $B_{j}$ is evaluated by the vector products of generated ruled surface contact vector $\left(\overrightarrow{k_{B j}}\right)$ and the normalized tangent vector $\left(\overrightarrow{t_{B j}}\right)$ of the bottom curve at $B_{j}$

$\overrightarrow{n_{B j}}=\overrightarrow{k_{B j}} \wedge \overrightarrow{t_{B j}} \rightarrow \overrightarrow{k_{B j}}=\frac{\overrightarrow{B_{j} T_{j}}}{\left\|\overrightarrow{B_{j} T_{j}}\right\|}$

Eqs. (1)-(4) define the coordinate and orientation of the tapered helical ball end mill when it is tangent to the ruled surface at point $B_{j}$.

\subsection{Intersection of tapered helical ball end mill and ruled surface}

The intersection of cutter and part along the tool path is evaluated in $\mathrm{ACIS}^{\odot}$ [14] solid modelling environment.
Tapered ball end mill is modelled as a Boolean combination of cone and sphere primitives. The surface is imported from the CAD system to ACIS environment by its boundary represented (BREP) model. Since the cutter location and orientation is modelled with respect to the coordinate center in Section 2.1, the engagement of cutter with the workpiece is evaluated using surface/surface intersection operations in ACIS. The ball end and cone primitives are intersected separately with the workpiece for each calculated tool location $\overrightarrow{O E_{j}}$ and $\vec{C}_{B j} C_{T j}$ as illustrated in Fig. 4, where the outer surface of the part is a ruled surface while the finish part is a straight plane. Although this example is given here in order to verify the method on the available three and a half axis machining center, the strategy is identical in five-axis milling where each cutter orientation is tangent along a straight line. This example is also practical because it leads to verify the validity of cutting coefficients from various cutter/ workpiece engagements while avoiding errors coming from the tool positioning stage. The intersection leads to complete identification of cutter/workpiece engagement boundaries along a given tool path.

For a given tool location, each slice can be intersected with the edges calculated previously. That gives some

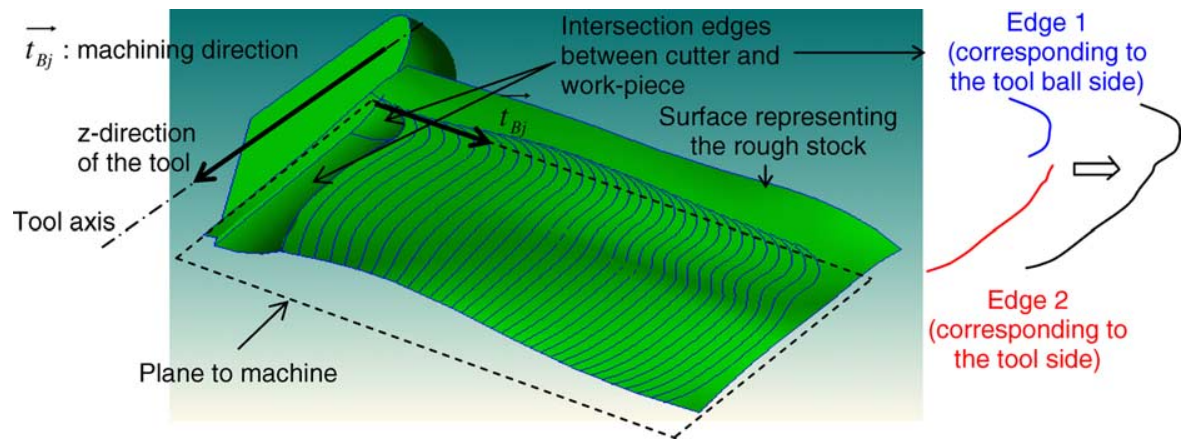

Fig. 4. Intersections between cutter surface and workpiece surface. 

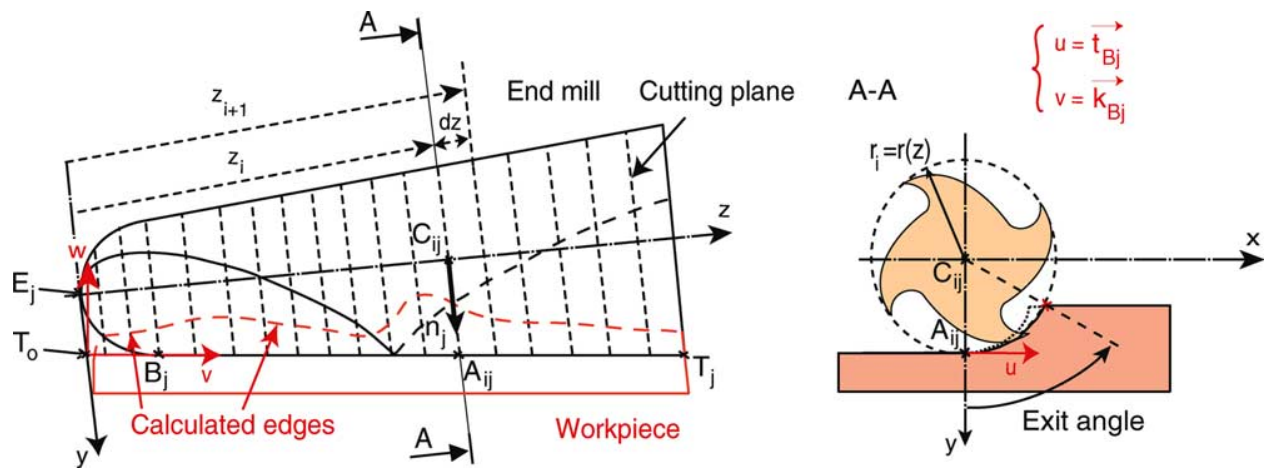

Fig. 5. Cutting planes definition and exit angle calculation.

intersection points. Entry and exit angles can therefore be determined along the $z$-direction of the tool.

\subsection{Evaluation of cutter immersion angles}

The aim of the geometric model is to identify cutter entry into and exit from the work material, which is required in predicting the cutting forces in flank milling operations. The tapered cutter is divided into a number of discrete disk elements along its axis, see Fig. 5. Each disk surface is considered as the plane of cut. At the boundary of each disk (i), the cutter cross-section is represented by a circle with varying radius $\left(r_{i}\right)$ along the cutter axis. The immersion at each elevation of the cutter axis, i.e. the entry $\left(\phi_{\text {start }}\right)$ and exit $\left(\phi_{\text {exit }}\right)$ angles need to be calculated. The cutter may intersect the workpiece with four possible scenarios as shown in Fig. 6.

Case 1. Cutter does not intersect the part.

Case 2. The cutter enters the workpiece with zero entry angle $\left(\phi_{\text {start }}\right)$ and exits at $\left(\phi_{\text {exit }}\right)$ in up milling, and the reverse is valid for down milling operations. The surface finish point $A_{i j}$ can be calculated by knowing the tool orientation from CL file or as formulated in Section 2.1

$\overrightarrow{O A}_{i j}=\overrightarrow{O C}_{i j}+r_{i} \cdot \vec{n}_{j}, \leftarrow \vec{n}_{j}=-\vec{t}_{B j} \wedge \vec{k}_{j}$

where $\overrightarrow{t_{B j}}$ is the unit feed vector along the tool path and $\vec{k}_{j}$ represents the tool axis, see Figs. 3, 5 and 6. The radius $r_{i}$ of the cutter disk element at elevation $z$ is given by (Fig. 5):

$$
\begin{array}{ll}
r_{i}=\left\|\overrightarrow{T_{0} A_{i j}}\right\| \cdot \sin (\gamma)+r_{\mathrm{b}} \cdot(1-\tan (\gamma)) & \text { if }\left\|\overrightarrow{T_{0} \vec{A}_{i j}}\right\| \geq r_{\mathrm{b}} \\
r_{i}=\sqrt{\left(r_{\mathrm{b}}^{2}-\left(r_{\mathrm{b}}-\left(\left\|{\overrightarrow{T_{0}}}_{i j}\right\| \cdot \cos (\gamma)\right)\right)^{2}\right)} & \text { if }\left\|\overrightarrow{T_{0} \vec{A}_{i j}}\right\|<r_{\mathrm{b}}
\end{array}
$$

Case 3. There are two intersection points on either sides of finish surface point $A_{i j}$. $P t_{1}$ is located on the right-side of $A_{i j}$ which is not in cut and tool starts with zero immersion angle, and $P t_{2}$ is on the left-side of $A_{i j}$ which gives the exit angle of the cut.

Case 4. The cutter starts with nonzero entry angle at $P t_{1}$, and exit at $P t_{2}$.

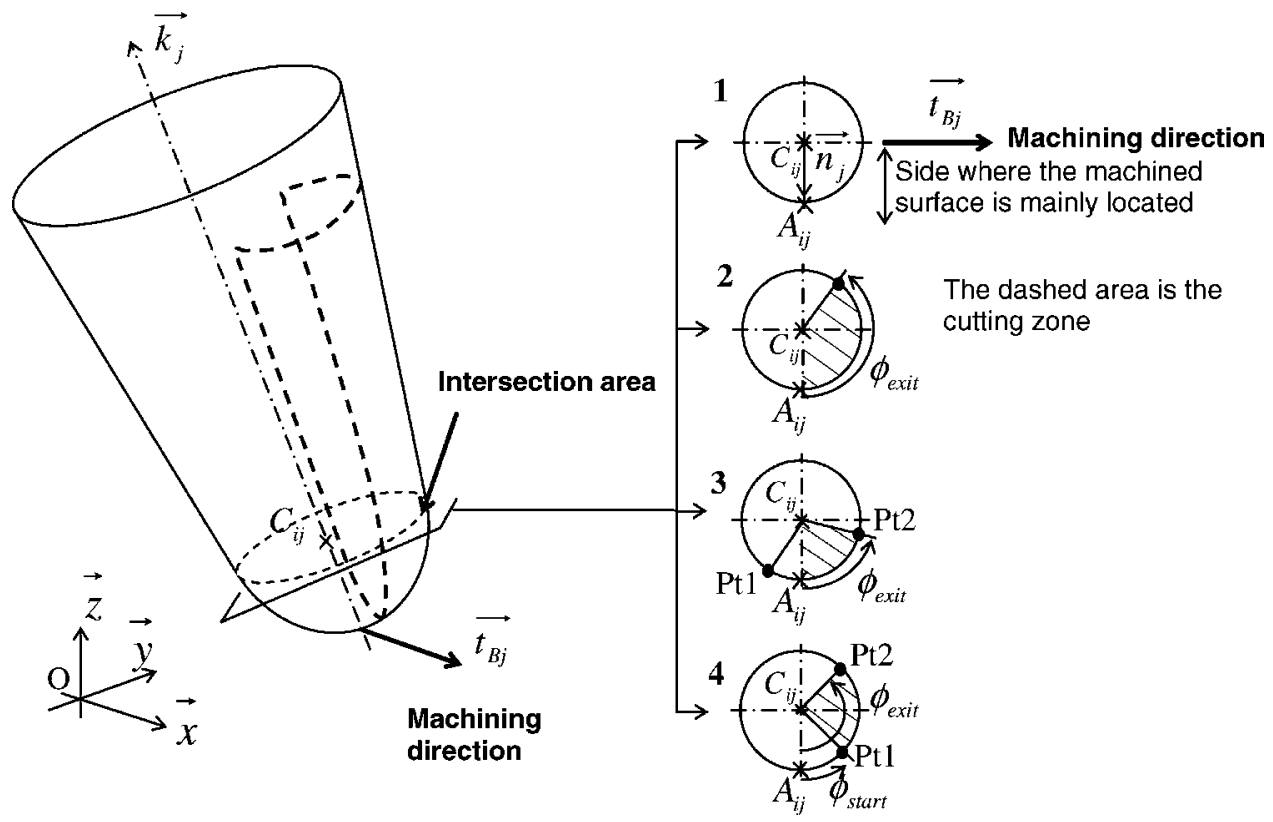

Fig. 6. Particularity of the engagement conditions in flank milling. 
Whether the immersion belongs to case 3 or 4 can be identified by evaluating the following vector relationships

$$
\left.\begin{array}{l}
\vec{V}_{1}=\vec{C}_{i j} A_{i j} \wedge \overrightarrow{C_{i j} P} t_{1}, \quad \vec{V}_{1} \cdot \vec{k}_{j} \\
\vec{V}_{2}=C_{i j} A_{i j} \wedge \overrightarrow{C_{i j} P} t_{2}, \quad \vec{V}_{2} \cdot \vec{k}_{j}
\end{array}\right\}
$$

where $\vec{V}_{1}$ and $\vec{V}_{2}$ represent whether the points lie on the left- or right-side of the surface finish point $A_{i j}$. If $\vec{V}_{1} \cdot \vec{k}_{j}$ has the same sign as $\vec{V}_{2} \cdot \vec{k}_{j}$, the intersection corresponds to case 4 , otherwise it is case 3 . The surface finish point $A_{i j}$ is calculated by offsetting circle center $C_{i j}$ at amplitude of radius $r_{i}$ in the direction perpendicular to the feed vector $\vec{t}_{B j}$. The immersion angles are calculated knowing $A_{i j}, C_{i j}$ and the intersection points $P t_{1}$ and $P t_{2}$.

For example, case 4 leads to verify that $\vec{V}_{1}$ and $\vec{V}_{2}$ have the same sign. As a result:

$$
\begin{aligned}
& \phi_{\text {start }}=\operatorname{angle}\left(\overrightarrow{C_{i j} A_{i j}}, \overrightarrow{C_{i j} P} t_{1}\right) \neq 0 \\
& \phi_{\text {exit }}=\operatorname{angle}\left(C_{i j} A_{i j}, \overrightarrow{C_{i j} P t_{2}}\right) \neq 0
\end{aligned}
$$

For case $3, \vec{V}_{1}$ and $\vec{V}_{2}$ have opposite signs. Then, immersion angles are:

$$
\begin{aligned}
& \phi_{\text {start }}=\operatorname{angle}\left(\overrightarrow{C_{i j}} \overrightarrow{A_{i j}}, \overrightarrow{C_{i j} P t_{1}}\right)=0 \\
& \phi_{\text {exit }}=\operatorname{angle}\left(\overrightarrow{C_{i j}} \overrightarrow{A_{i j}}, \overrightarrow{C_{i j} P} t_{2}\right) \neq 0
\end{aligned}
$$

The direction of spindle rotation, i.e. clock wise $(\mathrm{CW})$ or counter clock wise (CCW), is evaluated according to the logic shown in Fig. 7.

\subsection{Interpolation of cutter engagement}

A new coordinate system $\left(T_{O}, u, v, w\right)$ has been introduced to display the immersion angles along the considered tool path (Fig. 5). $u$ represents $\overrightarrow{t_{B j}}, v$ is $\overrightarrow{k_{B j}}$, where $\overrightarrow{t_{B j}}$ and $\overrightarrow{k_{B j}}$ represent vectors tangent to cutter periphery in radial and axial directions, respectively. $T_{O}$ is the projection of cutter periphery on $y$-axis and evaluated as follows:

$\overrightarrow{O T}_{o}=\overrightarrow{O E}_{j}+r_{\mathrm{b}} \cdot(1-\tan (\gamma)) \cdot \vec{y}, \leftarrow \vec{y}=\vec{n}_{j}$

Therefore along a given tool path, two immersion angles maps representatives of the angular variation of entry and exit angles have been calculated. Fig. 8a illustrates the calculation of intersection points for the previous example. Planes of cut are here represented with circles. Fig. 8b represents the evolution of exit angle along the tool path. In this example, entry angles are always equal to zero.

The immersion angles cards lead to define the most constraint zone on the part to be machined because tool load results from the difference between exit and entry angles. The interpretation of the card can lead either to a re-design of the part or to an optimization of the tool path on critical zones.

Once the immersion angles tables are obtained, the cutting forces simulation can be performed for each tool location. Cutting forces calculation requires to discretize the tool axially and has to be done for every tool locations along the motion according to feedrate requirements.

The cutter/part intersection and the resulting immersion angles are first calculated with large increments in ACIS solid modeling environment, which has high computational cost. As a result, feed per tooth increments are not taken into account.

It is proposed that the immersion angles are calculated at large discrete intervals along the tool path in ACIS, while not causing significant deviations from one station to the next. Then, the resulting immersion angles are interpolated later at finer feed intervals, i.e. feed per tooth increments, using the following proposed method.

The new discretization distance pitch_dis has to be calculated. First tool location is assumed to correspond to $u=0$ and last location to $u=1$. By calculating the curvilinear abscissa Abcurv in $u$-direction between the first and last tool locations, it is possible to calculate the approximate number of tool rotations necessary to machine the impeller $\left(\left(\right.\right.$ floor $\left.\left(A b \operatorname{curv}_{x} / f z \cdot N_{\mathrm{f}}\right)\right)$. Consequently, we know the interval

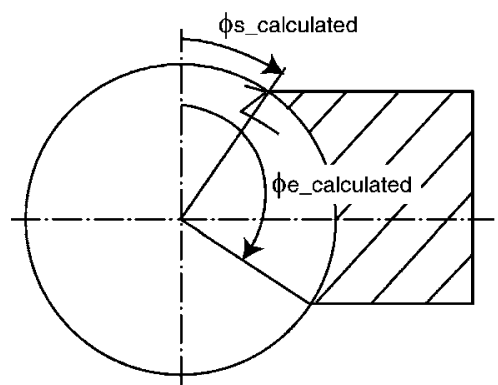

$|\phi e x i t|=\mid \phi e_{-}$calculated $\mid$

$|\phi s t a r t|=\mid \phi s \_$calculated $\mid$

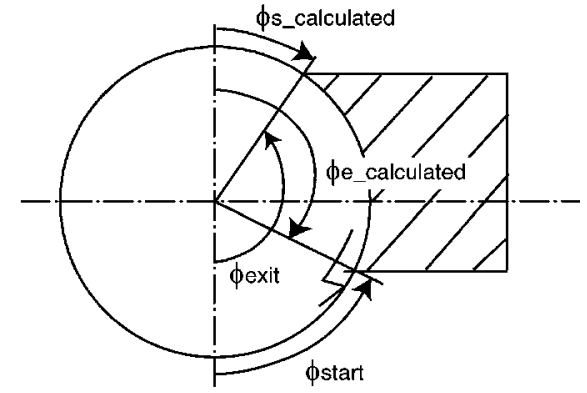

$\mid \phi$ exit $|=\pi-| \phi s_{-}$calculated $\mid$

$\mid \phi$ start $|=\pi-| \phi e_{-}$calculated $\mid$

Fig. 7. Taking into account of the direction of the cutting motion. 
(a)

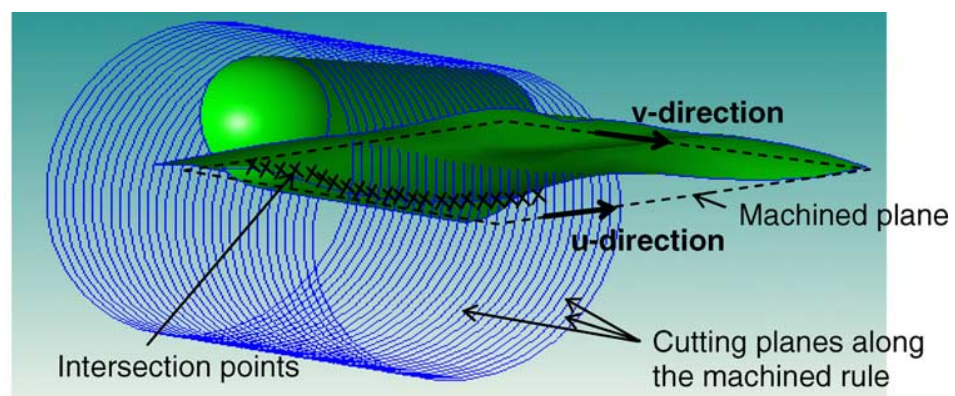

(b)

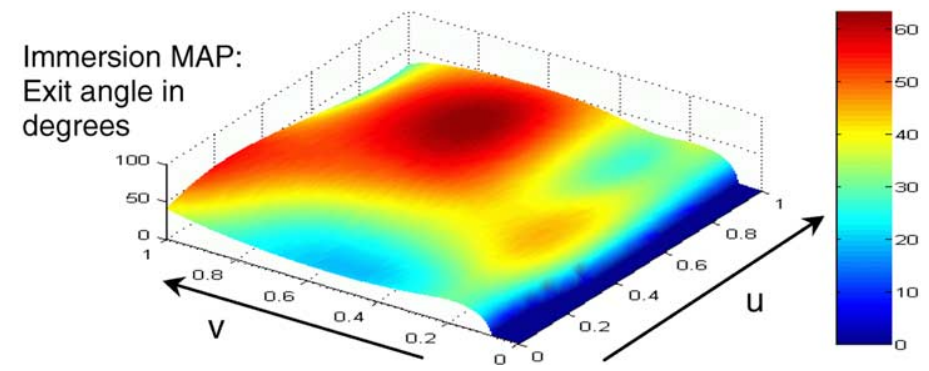

Fig. 8. Various immersion angles calculation.

in $u$-direction between two tool locations:

pitch_dis $=1 /$ floor $\left(\frac{A b c u r v_{x}}{f_{z} \cdot N_{\mathrm{f}}}\right)$

Then, we take advantage of the bi-parametric representation of the immersion map $(u, v)$ by interpolating a new grid of angles considering pitch_dis. Interpolation step has been performed in Matlab ${ }^{\mathbb{C}}$ software [15] through the use of a function dealing with data interpolation. This function is based on a Delaunay triangulation of the data that uses Qhull method (see http://www.qhull.org/, June 2004).

\section{Mechanics of milling with tapered helical ball end mill}

The mechanics of tapered helical ball end mill for slot milling operations have been presented before by Engin and Altintas [12], as well as by Ramaraj and Eleftheriou [10] in the past. Hence, only the extension of mechanics in flank milling with varying engagement conditions, and optimization of feedrate to constrain cutting forces will be presented here.

The tapered cutter is divided into a number of discrete disk elements as explained in immersion angle calculation. The model assumes that the $\vec{x}$-axis $\left(\vec{x}=\overrightarrow{t_{B j}}=u\right)$ is always aligned with the feed direction, the normal to the feed or surface finish is $\vec{y}\left(\vec{y}=\vec{n}_{j}\right)$, and the cutter axis is in $\vec{z}$ direction $(\vec{z}=\vec{x} \wedge \vec{y})$. The tool tip is at point $E_{j}$, which is used as a reference.

At any cutter disc element, differential cutting forces in tangential $\left(F_{\mathrm{t} p}\right)$, radial $\left(F_{\mathrm{r} p}\right)$ and axial $\left(F_{\mathrm{a} p}\right)$ directions act on each tooth $(p)$ which is within the cut or immersion zone (Fig. 9)

$$
\begin{aligned}
& \mathrm{d} F_{\mathrm{t}}=K_{\mathrm{te}} \mathrm{d} S+K_{\mathrm{tc}} f_{z} \cdot \sin \left(\phi_{p}\right) \cdot \sin (\kappa) \mathrm{d} b \\
& \left.\mathrm{~d} F_{\mathrm{r}}=K_{\mathrm{re}} \mathrm{d} S+K_{\mathrm{rc}} f_{z} \cdot \sin \left(\phi_{p}\right) \cdot \sin (\kappa) \mathrm{d} b\right\} \\
& \mathrm{d} F_{\mathrm{a}}=K_{\mathrm{ae}} \mathrm{d} S+K_{\mathrm{ac}} f_{z} \cdot \sin \left(\phi_{p}\right) \cdot \sin (\kappa) \mathrm{d} b
\end{aligned}
$$
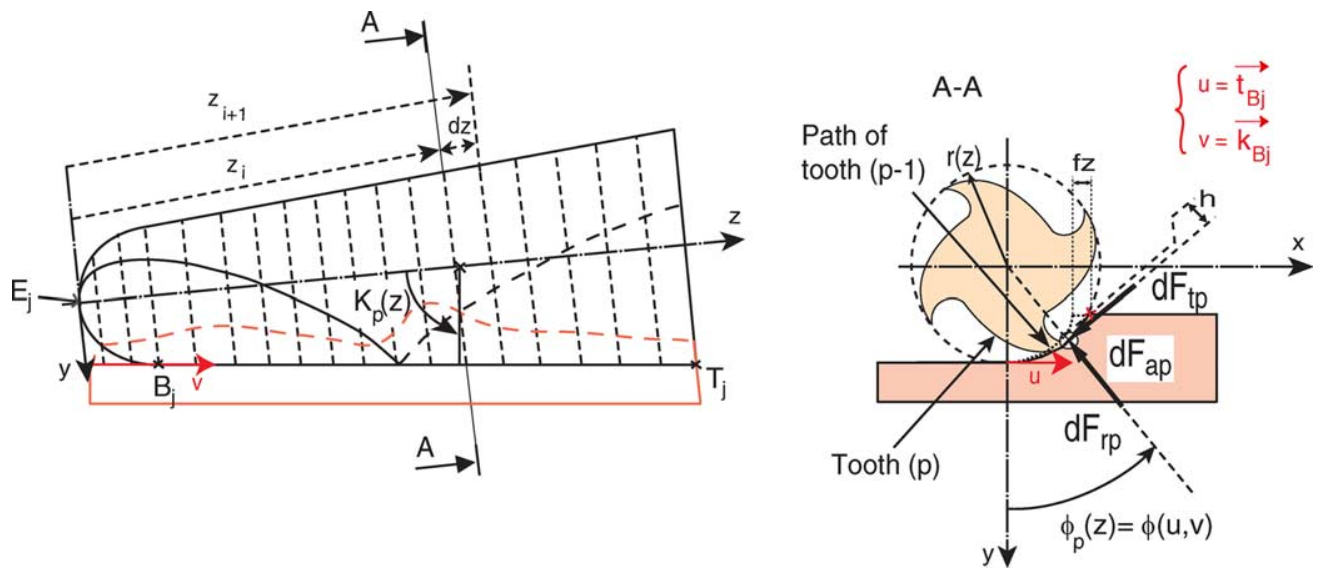

Fig. 9. Cutting forces calculation. 


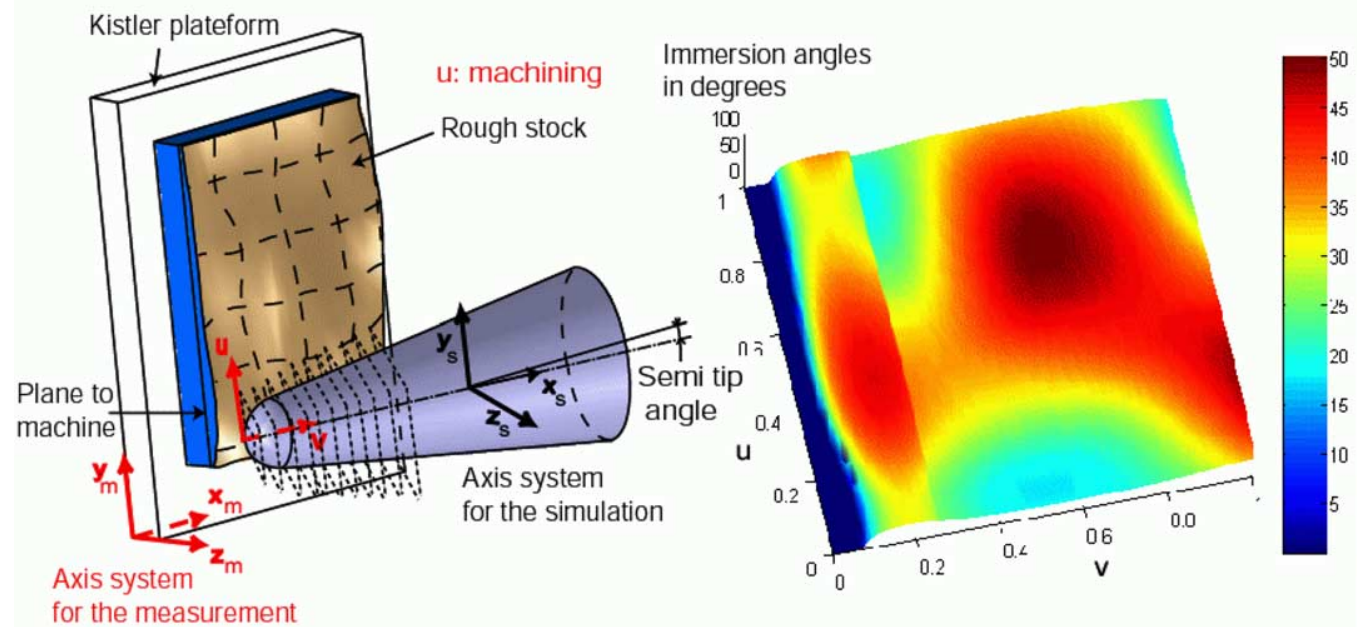

Fig. 10. Cutting forces measurement during the machining.

where $f_{\mathrm{z}}$ is the feed per tooth, $\mathrm{d} b=\mathrm{d} z / \sin \kappa$ and $\mathrm{d} z$ is the axial disk element height, $\gamma$ is the half taper angle of the cutter, $\phi_{p}$ is the instantaneous immersion angle of disk element $i$ which belongs to flute $p$ [16]. The differential cutting edge contact length is $\mathrm{d} S$ and given as $\left(\mathrm{d} S=|\mathrm{d} r|=\sqrt{r^{2}(\phi)+\left(r^{\prime}(\phi)\right)^{2}+\left(z^{\prime}(\phi)\right)^{2}} \cdot \mathrm{d} \phi, r^{\prime}(\phi)=\mathrm{d}\right.$ $\left.r(\phi) / \mathrm{d} \phi, z^{\prime}(\phi)=\mathrm{d} z(\phi) / \mathrm{d} \phi\right)$ by Engin and Altintas [12]. Note that the flute cuts and produces cutting force only when it is immersion zone, e.g. $\phi_{\text {start }} \leq \phi_{p} \leq \phi_{\text {exit }}$. The cutting force coefficients $K_{\mathrm{tc}}, K_{\mathrm{rc}}, K_{\mathrm{ac}}, K_{\mathrm{te}}, K_{\mathrm{re}}, K_{\mathrm{ae}}$ are found either from the material orthogonal cutting data base, or from mechanistic calibration of specific tapered helical end mill as described in $[17,18]$. The immersion angles may be different at each segment along the cutter axis, but they are calculated in CAD model and stored in an engagement data file which is used as an input by the force prediction and optimization module. The differential forces for each cutter segment along the cutter axis are calculated, projected in three directions $(\vec{x}, \vec{y}, \vec{z})$,

$$
\begin{aligned}
{\left[\begin{array}{l}
\mathrm{d} F_{x p} \\
\mathrm{~d} F_{y p} \\
\mathrm{~d} F_{z p}
\end{array}\right]=} & {\left[\begin{array}{ccc}
-\sin \phi_{p} \sin \kappa_{p} & -\cos \phi_{p} & -\sin \phi_{p} \cos \kappa_{p} \\
-\cos \phi_{p} \sin \kappa_{p} & \sin \phi_{p} & -\cos \phi_{p} \cos \kappa_{p} \\
-\cos \kappa_{p} & 0 & -\sin \kappa_{p}
\end{array}\right] } \\
& \times\left[\begin{array}{l}
\mathrm{d} F_{\mathrm{r} p} \\
\mathrm{~d} F_{\mathrm{t} p} \\
\mathrm{~d} F_{\mathrm{a} p}
\end{array}\right]
\end{aligned}
$$

and summed to find total feed $(x)$, normal $(y)$ and axial (z) cutting forces acting on the cutter

$$
\begin{aligned}
& F_{x}(\phi)=\sum_{p=1}^{N_{\mathrm{f}}} F_{x p}\left[\phi_{p}(z)\right]=\sum_{p=1}^{N_{\mathrm{f}}} \sum_{i=1}^{N_{i}} \int_{z_{i}}^{z_{i+1}}\left[\mathrm{~d} F_{x p}\right] \mathrm{d} z \\
& F_{y}(\phi)=\sum_{p=1}^{N_{\mathrm{f}}} F_{y p}\left[\phi_{p}(z)\right]=\sum_{p=1}^{N_{f}} \sum_{i=1}^{N_{i}} \int_{z_{i}}^{z_{i+1}}\left[\mathrm{~d} F_{y p}\right] \mathrm{d} z \\
& F_{z}(\phi)=\sum_{p=1}^{N_{\mathrm{f}}} F_{z p}\left[\phi_{p}(z)\right]=\sum_{p=1}^{N_{\mathrm{f}}} \sum_{i=1}^{N_{i}} \int_{z_{i}}^{z_{i+1}}\left[\mathrm{~d} F_{z p}\right] \mathrm{d} z
\end{aligned}
$$
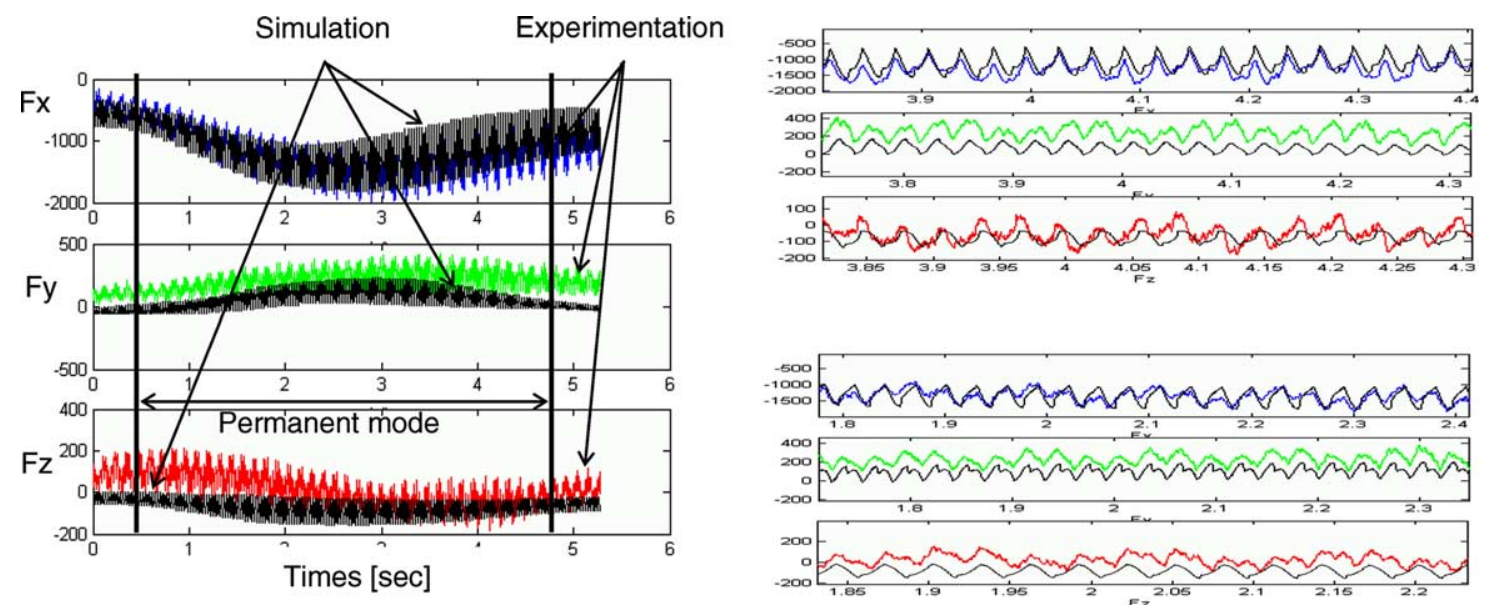

Fig. 11. Comparison between simulated and measured cutting forces. 

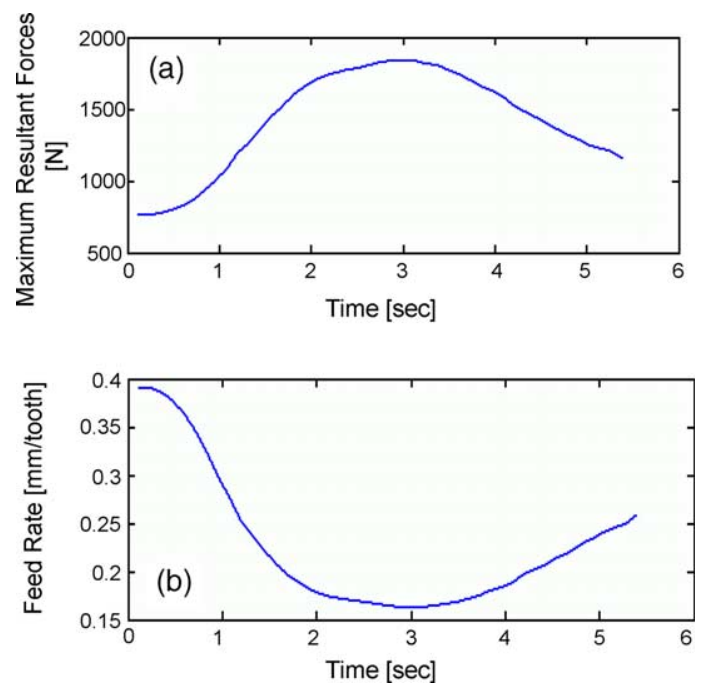

Fig. 12. Feedrate scheduling.

where

- $N_{\mathrm{f}}$ is the number of flutes on the cutter,

- $N_{i}$ is the number of discrete cutter elements in $z$ direction,

- $z_{i}$ and $z_{i+1}$ are the contact boundaries of cutter disk element,

- $\phi_{p}=\Omega \cdot t$ is the angular position of flute $p$ and disk element $i$, and $\Omega$ is the angular speed of the spindle.

The differential cutting forces are integrated along the full contact length for all flutes which are engaged. For
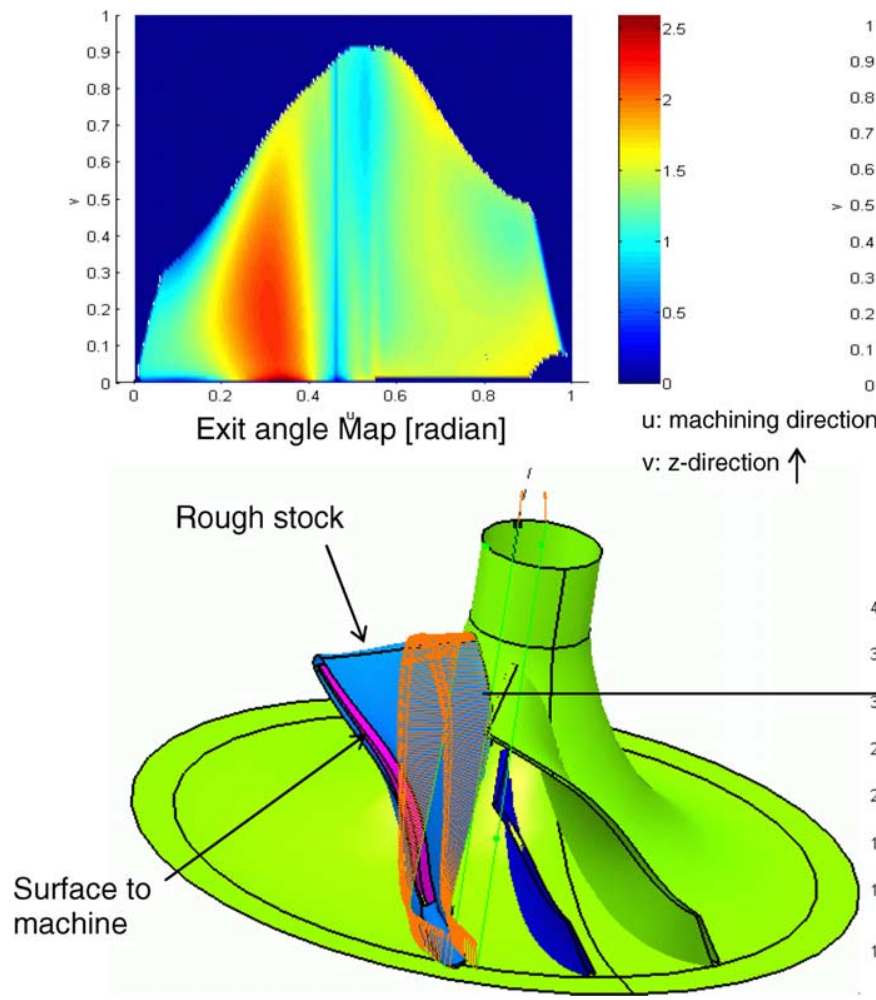

$\mathrm{u}:$ machining direction $\rightarrow \quad$ Start ăngle Map [radian]

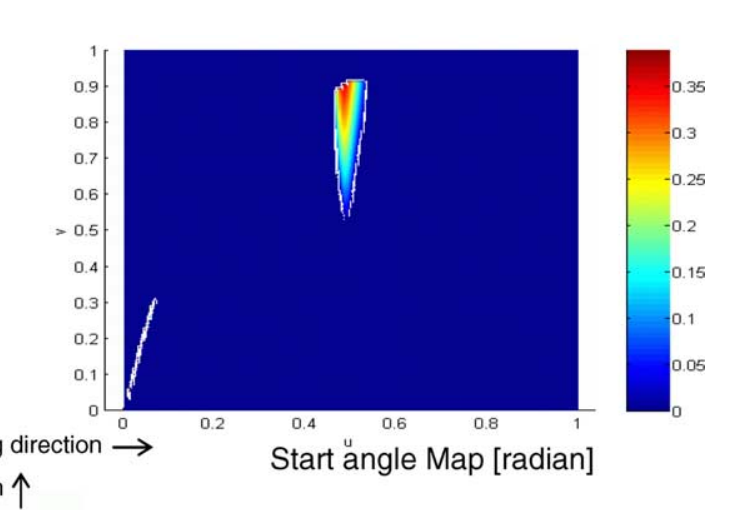

The proposed simulation of flank milling process is experimentally verified on existing three and a half axis horizontal CNC (Mori Seiki HS 402) machining center. As presented in Section 2, the algorithm covers five-axis flank milling process simulation as well, but a five-axis machine was not available in the laboratory for cutting tests. Aluminum AL7075-T6 was prepared with a ruled outer surface as shown in Fig. 10. The cutter was a tapered helical ball end mill with $6.35 \mathrm{~mm}$ ball radius, $6.25^{\circ}$ half taper angle and four flutes. The cutter/ruled surface intersections are calculated for up milling mode and immersion angles are stored in a file. The identified immersion angle history is shown in Fig. 10.

The first test was conducted at a constant feedrate $f_{\mathrm{z}}=0.2 \mathrm{~mm} /$ tooth per rev. The spindle speed was

Fig. 13. Immersion cards for the machining of an impeller surface. 


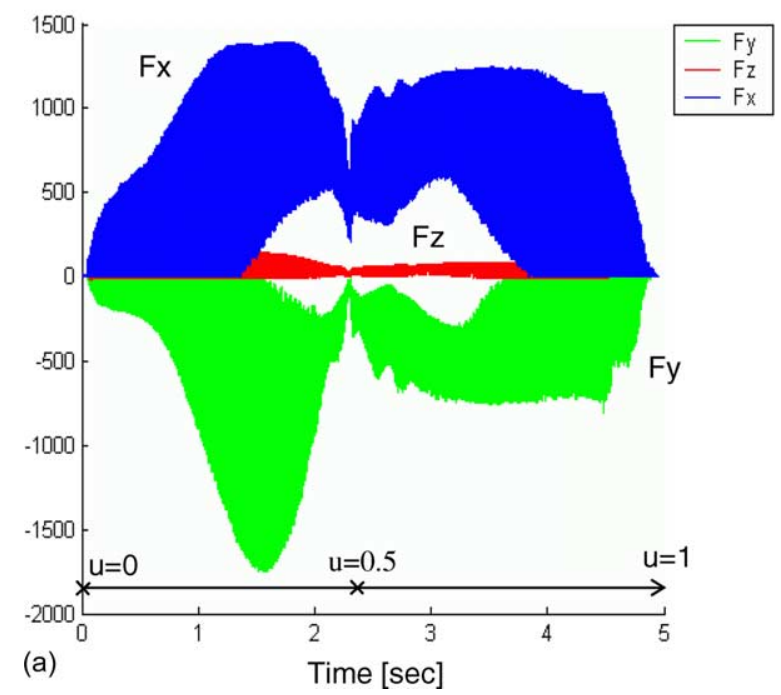

(b)

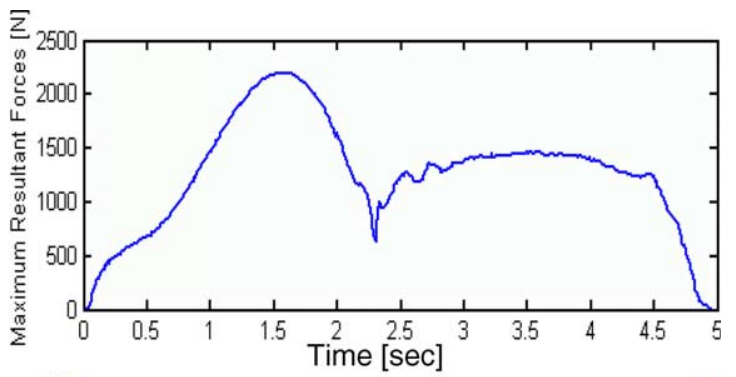

Fig. 14. Virtual scheduling of feedrate during five-axis machining of an impeller surface.

$500 \mathrm{rev} / \mathrm{min}$. The simulated and experimental measured cutting force history and a sample close up window of data are shown in Fig. 11. Although prediction accuracy is reasonable, there are some deviations in the normal $(\vec{y})$ and axial $(\vec{z})$ directions which are mainly due to errors in cutting force coefficients and static deflection of the tool in radial direction which changes the effective immersion angle, hence the chip loads.

When the feed is kept constant at $f_{\mathrm{z}}=0.2 \mathrm{~mm} /$ tooth, the maximum resultant force varies along the tool path as predicted in Fig. 12a. Impellers are usually made from thermally resistant materials such as Titanium and Nickel alloys, and they produce large forces and heat which may break the tool. It is preferred to maintain the cutting force at a safe and desired level so that the cutter is not broken and tool-blade deflections do not violate the tolerance of the part. Using the force prediction algorithm, the feed is scheduled along the tool path so that the resultant force remains at $1500 \mathrm{~N}$ regardless of the changes in the cutter engagement conditions along the tool path. The evaluated feedrate history for constant force is given in Fig. 12b.

Although it was not possible to test milling of impeller due to lack of five-axis machining center, Fig. 13 shows a sample simulation. The predicted cutting forces along the finish machining of impeller blade is shown in Fig. 14. The feedrate was $0.1 \mathrm{~mm} /$ tooth, and the tool geometry was the same as in previous test. The resultant force is shown in Fig. 14b when the feed is constant at $c=0.1 \mathrm{~mm} /$ tooth. The feedrate is scheduled to maintain the maximum force, torque and power were constrained at $2500 \mathrm{~N}, 2000 \mathrm{~N} \mathrm{~mm}$ and $50 \mathrm{~kW}$, respectively. The resulting feed profile is given in Fig. 14c.

\section{Conclusion}

Simulation model for flank milling processes is presented. The part/cutter intersection boundaries are evaluated using the geometric models of the tapered helical ball end mill and ruled surface to be machined. The cutter immersion angles are extracted from the geometric model, and used as boundary conditions in predicting three to five-axis flank milling operations with tapered helical ball end mills. The cutting forces are predicted, and compared favorably with the measured cutting forces. The model allows scheduling of the feedrate along the complex tool path so that the cutting forces acting on the cutter and blade are kept at the desired but also at productive levels. The model can be used for both three- and five-axis flank milling operations where the final- and semi-finished workpiece geometry is known.

\section{Acknowledgements}

This research has been supported by National Science and Research Council of Canada and Pratt \& Whitney Canada as part of Virtual Machining Research Chair.

\section{References}

[1] C.Y. Wu, Arbitrary surface flank milling of fan, compressor and impeller blades, Transactions of the ASME, Journal of Engineering for Gas Turbines and Power 117 (1995) 534-539.

[2] C.Y. Wu, Y. Altintas, R.A. Thompson, Tool positioning and feedrate problems in impeller flanks milling, Proceedings of the Canadian Conference on Industrial Computer System, McMaster University, Hamilton, Ont., Canada 1982; 13.1-13.6. 
[3] J.C.J. Chiou, Accurate tool position for 5-axis rule surface machining by swept envelope approach, Computer Aided Design 36 (10) (2004) 967-974.

[4] J.C.J. Chiou, Y.S. Lee, Swept surface determination for 5-axis numerical control machining, International Journal of Machine Tools and manufacture 42 (2002) 1497-1507.

[5] J.C.J. Chiou, Y.S. Lee, A shape-generating approach for multiaxis machining G-buffer models, Computer Aided Design 31 (1999) 761-776

[6] C. Lartigue, E. Duc, A. Affouard, Tool path deformation in 5-axis flank milling using envelope surface, Computer Aided Design 35 (4) (2003) 375-382.

[7] H. Tonshoff, Optimal tool positioning for 5-axis milling of arbitrary shaped surfaces, Production Engineering 7 (1) (2000).

[8] F. Monies, J.M. Redonnet, W. Rubio, P. Lagarrigue, Improved positioning of a conical mill for machining ruled surfaces, Journal of Engineering Manufacture 214 (7) (2000) 625-634.

[9] Y. Altintas, Manufacturing Automation, Metal Cutting Mechanics, Machine Tools Vibrations and CNC Design, Cambridge University Press, Cambridge, MA, 2000.

[10] T.C. Ramaraj, E. Eleftheriou, Analysis of the mechanics of machining with tapered end milling cutters, Transactions of ASME 116 (1994) 398-404.
[11] Y. Altintas, P. Lee, A general mechanics and dynamics model for helical end mills, Annals of the CIRP 45 (1) (1996) 59-64.

[12] S. Engin, Y. Altintas, Modeling of general milling operations: Part I. End mills, International Journal of Machine Tools and Manufacture 41 (2001) 2195-2212.

[13] E. Budak, Improvement of productivity and part quality in milling of titanium based impellers by chatter suppression and force control, Annals of the CIRP 49 (1) (2000) 31-36.

[14] 3D ACIS ${ }^{\odot}$ Modeler, Spatial's prominent 3D modeling development technology, http://www.spatial.com/products/3D/modeling/ (June 2004).

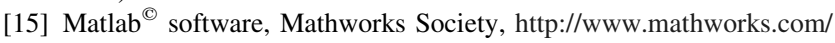
(June 2004).

[16] Y. Altintas, P. Lee, Mechanics and dynamics of ball end milling, Transactions of the ASME, Journal of Manufacturing Science and Engineering 120 (1998) 684-692.

[17] E. Budak, Y. Altintas, E.J.A. Armarego, Prediction of milling force coefficients from orthogonal cutting data, Transactions of ASME 118 (1996) 216-224.

[18] J. Gradisek, M. Kalveram, K. Weinert, Mechanistic identification of specific force coefficients for a general end mill, International Journal of Machine Tools and Manufacture 44 (2004) 401-414. 